\title{
COMPARISON THEOREM FOR CONJUGATE POINTS OF SYSTEMS OF $n$ TH ORDER NONSELFADJOINT DIFFERENTIAL EQUATIONS
}

\author{
E. C. TOMASTIK
}

\begin{abstract}
A comparison theorem for conjugate points of the two systems of linear differential equations $x^{(n)}-(-1)^{n-k} p(t) x=0$ and $y^{(n)}-$ $(-1)^{n-k} q(t) y=0$, where $p(t)$ and $q(t)$ are $m \times m$ matrices of continuous functions, is given. It is assumed that $q(t)$ is positive with respect to a certain cone but no positivity conditions of any kind are imposed on $p(t)$. No selfadjointness conditions are assumed; however, the results are new even in the selfadjoint case.
\end{abstract}

This paper shall be concerned with the comparison of the conjugate points of the two systems of linear differential equations

$$
x^{(n)}-(-1)^{n-k} p(t) x=0
$$

and

$$
y^{(n)}-(-1)^{n-k} q(t) y=0
$$

where $p(t)$ and $q(t)$ are $m \times m$ matrices of continuous functions on some interval $[a, b], a \geq 0$. As will be seen later, $q(t)$ will satisfy a "positivity" condition with respect to a certain cone. But no positivity conditions of any type whatsoever are placed on the matrix $p(t)$. In particular, $p(t)$ could have oscillatory components. Since no assumptions are made concerning the symmetry of the matrices $p(t)$ and $q(t)$ nor concerning the integers $n$ and $k$, systems (1) and (2) will in general be nonselfadjoint. But even in the case that both (1) and (2) are selfadjoint, the results presented here are new. The results are also new even if $n=2$.

The first comparison theorem for second order systems was given by Morse [6]. Morse assumed both systems (1) and (2) were selfadjoint, i.e., $p(t)$ and $q(t)$ were symmetric. He showed that the first conjugate point of (1) lies to the right of the first conjugate point of (2) if $q(t)-p(t)$ is positive semidefinite everywhere and positive definite at at least one point. Reid $[8$, p. 356] extends these results to selfadjoint systems of order $2 n$.

More recently, a number of individuals have given comparison theorems for (1) and (2) in the nonselfadjoint case and for which the results are new even if (1) and (2) are selfadjoint. The first such results have been given by Cheng [3], Schmitt and Smith [9], Smith [10], Keener and Travis [4], and the author [11]. All of these papers except Cheng and Keener and Travis are for $n=2$. Also, all of these papers except for author's [11], assume that $p(t)$ and $q(t)$ satisfy certain strong positivity

Received by the editors September 4, 1984.

1980 Mathematics Subject Classification. Primary 34C10; Secondary 47B55, 47E05.

Key words and phrases. Conjugate point, comparison theorem, $\mu_{0}$-positive operators with respect to a cone. 
conditions, whereas in this paper no positivity conditions whatsoever are placed on $p(t)$.

In this paper it is assumed that some partition $\{I, J\}$ of the integers $\{1,2, \ldots, m\}$ has been given, that is, $I \cup J=\{1, \ldots, m\}$ and $I \cap J=\varnothing$. The cone $K$ is then defined by

$$
K=\left\{\left(z_{1}, \ldots, z_{m}\right): \mu \in I \Rightarrow z_{\mu} \geq 0, \mu \in J \Rightarrow z_{\mu} \leq 0\right\} .
$$

The interior of $K$ is defind to be $K^{\circ}$.

Throughout this paper it is assumed that for every $t \in[a, b], v \in K$ and $v \neq 0$, $q(t) v \in K^{\circ}$.

Two such examples are given by

$$
\left[\begin{array}{ll}
1 & 1 \\
2 & 1
\end{array}\right] \text { and }\left[\begin{array}{cc}
1 & -1 \\
-2 & 1
\end{array}\right]
$$

For the first map $K$ is the cone given by the first quadrant, and for the second map $K$ is the cone given by the fourth quadrant.

The number $c_{p}(a)=b$ is the first conjugate point of (1) if (1) has a solution $x(t)$ not identically zero such that $x^{(i)}(a)=0, i=0,1, \ldots, k-1$, and $x^{(i)}(b)=0$, $i=0,1, \ldots, n-k-1$, and (1) has no other solution $\bar{x}(t)$ not identically zero such that $\bar{x}^{(i)}(a)=0, i=0,1, \ldots, k-1$, and $\bar{x}^{(i)}(\beta)=0, i=0, \ldots, m-k-1$, where $a<\beta<c_{p}(a)$.

Instead of dealing with (1) directly, a certain equivalent integral equation will be considered. This integral equation uses a Green's function that has been put in an appropriate form for the analysis that is to follows. Toward this end define

$$
G(v, u)=\frac{1}{(n-k-1) !(k-1) !} \int_{0}^{\delta}(v-\xi)^{k-1}(u-\xi)^{n-k-1} d \xi, \quad \delta=\min u, v
$$

and

$$
g(t, s, \alpha, \beta)=G((t-\alpha)(\beta-s) /(\beta-\alpha),(s-\alpha)(\beta-t) /(\beta-\alpha)) .
$$

Nehari [7] and Travis [12] have already established that $g(t, s, \alpha, \beta)$ is the Green's function for

$$
\begin{aligned}
& (-1)^{n-k} y^{(n)}=0, \quad y^{(i)}(\alpha)=0, \quad i=0, \ldots, k-1, \\
& y^{(i)}(\beta)=0, \quad i=0, \ldots, n-k-1 .
\end{aligned}
$$

Thus $x(t)$ is a solution of $(1)$ with $x^{(i)}(\alpha)=0, i=0, \ldots, k-1, x^{(i)}(\beta)=0$, $i=0, \ldots, n-k-1$, if and only if

$$
x(t)=\int_{\alpha}^{\beta} g(t, s, \alpha, \beta) p(s) x(s) d s .
$$

Before establishing an important theorem, the following lemma is recalled from Keener and Travis [5].

LEMMA 1. Suppose $f:[\alpha, \beta] \rightarrow K$ is continuous and $f(t) \in K^{\circ}$ for some $t \in[\alpha, \beta]$. Then $\int_{\alpha}^{\beta} f(s) d s \in K^{\circ}$.

THEOREM 1. If the first conjugate point of (2) is $c_{q}(a)=b$, then (2) has a solution $x(t)$ such that $x^{(i)}(a)=0, i=0, \ldots, k-1$, and $x^{(i)}(b)=0, i=$ $0, \ldots, n-k-1$, and $x(t) \in K^{\circ}$ for all $t \in(a, b)$. 
PROOF. To establish this theorem define the Banach space

$$
B=\left\{u \in C^{n-1}([a, b]): u^{(i)}(a)=0, i=0, \ldots, k-1\right\},
$$

with norm $\|u\|=\sup \{|u(t)|: t \in[a, b]\}$. Also define the cone

$$
\tilde{K}=\{u \in B: u(t) \in K \text { for } t \in[a, b]\},
$$

with interior

$$
\tilde{K}^{\circ}=\left\{u \in B: u(t) \in K^{\circ} \text { for } t \in(a, b)\right\} .
$$

Consider the operator

$$
T(u)=\int_{a}^{b} g(t, s, a, b) q(s) u(s) d s .
$$

Exactly as in [4] it follows that $T$ maps $\tilde{K}$ into $\tilde{K}^{\circ}$ and therefore by arguments similar to those found in [4], $T$ is $\mu_{0}$-positive with respect to the cone $\tilde{K}$. From this, Theorem 1 follows in the same way as found in [4].

It is useful at this time to give without a proof a result that characterizes the structure of the matrix $q=\left(q_{i j}\right)$.

LEMMA 2. If $q v \in K^{\circ}$ for all $v \in K, v \neq 0$, then $q_{i j} \neq 0$ and $q_{i j}=\left|q_{i j}\right| \delta_{i} \delta_{j}$, where $\delta_{\mu}=1$ if $\mu \in I$ and $\delta_{\mu}=-1$ if $\mu \in J$.

Given any $\alpha \in[a, b), c_{p}(\alpha)$ and $c_{q}(\alpha)$ will be the first conjugate points of $\alpha$ of (1) and (2) respectively. The main theorem can now be given.

THEOREM 2. Suppose $\alpha \in[a, b), c_{q}(a)=b$ and $\left|p_{i j}(t)\right| \leq\left|q_{i j}(t)\right|$ for all $t \in[a, b]$ and all $i, j=1, \ldots, m$, and given any $i=1, \ldots, m$, there exists $j_{i} \in\{1, \ldots, m\}$ and $\tau_{i} \in[a, b]$ such that $\left|p_{i j_{i}}\left(\tau_{i}\right)\right|<\left|q_{i j_{i}}\left(\tau_{i}\right)\right|$. Then $c_{p}(\alpha)>c_{q}(a)$.

PROOF. Suppose contrary to the conclusion of Theorem 2, that $x(t)$ is a nontrivial solution of (1) satisfying the boundary conditions $x^{(i)}(\alpha)=0, i=0, \ldots, k-1$, and $x^{(i)}(\beta)=0, i=0, \ldots, n-k-1$, for some $\alpha, \beta \in[a, b], \alpha<\beta$. Then of course

$$
x(t)=\int_{\alpha}^{\beta} g(t, s, \alpha, \beta) p(s) x(x) d s .
$$

From Theorem 1, there exists a nontrivial solution $y$ of $(2)$ such that $y^{(i)}(a)=0$, $i=0, \ldots, k-1, y^{(i)}(b)=0, i=0, \ldots, n-k-1$, and

$$
y(t)=\int_{a}^{b} g(t, s, a, b) q(s) y(s) d s .
$$

Let $y=\left(y_{i}\right)$. It will now be shown that for any $i, y_{i}^{(k)}(a) \neq 0, y_{i}^{(n-k)}(b) \neq 0$. Now

$$
y^{(k)}(a)=\int_{a}^{b} \frac{\partial^{k}}{\partial t^{k}} g(a, s, a, b) q(s) y(s) d s .
$$

Using the definition of $g$, it follows that

$$
\frac{\partial^{k}}{\partial t^{k}} g(a, s, a, b)=\frac{1}{(n-k-1)}(s-a)^{n-k-1}\left(\frac{b-s}{b-a}\right)^{k} \text {. }
$$

What is important here is that $\left(\partial^{k} / \partial t^{k}\right) g(a, s, a, b)$ is positive for $s \in(a, b)$. From Theorem $1, y(s) \in K$ for $s \in[a, b]$ and therefore $q(s) y(s) \in K^{\circ}$ for $s \in(a, b)$. 
From the comments above concerning $\left(\partial^{k} / \partial t^{k}\right) g(a, s, a, b)$ and Lemma 1 , it follows that the right-hand side of $(3)$ is in $K^{\circ}$. This means from (3) that $y^{(k)}(a) \in K^{\circ}$ and this implies that $y_{i}^{(k)}(a) \neq 0$. One can show in a very similar manner that $y_{i}^{(n-k)}(b) \neq 0$.

Thus $y_{i}(t)$ has a zero at $t=\alpha$ of at most $k$ and a zero at $t=\beta$ of at most $n-k$. Of course $x_{i}(t)$ has a zero at $t=\alpha$ of at least $k$ and a zero at $t=\beta$ of at least $n-k$. Thus the terms $\left|x_{i}(t)\right| /\left|y_{i}(t)\right|$ are continuous on $(\alpha, \beta)$ and most importantly bounded on $(\alpha, \beta)$. Define

$$
\left\|x_{i}\right\|=\sup \left\{\left|x_{i}(t)\right| /\left|y_{i}(t)\right|: t \in(\alpha, \beta)\right\}
$$

and

$$
\|x\|=\max \left\{\left\|x_{i}\right\|: i=1, \ldots, m\right\} .
$$

In [7], Nehari has shown that for $a \leq \alpha \leq \beta \leq b, g(t, s, \alpha, \beta) \leq g(t, s, a, b)$. For $t \in(\alpha, \beta)$, it readily follows that

$$
\begin{aligned}
\left|x_{i}(t)\right| & =\left|\sum_{j} \int_{\alpha}^{\beta} g(t, s, \alpha, \beta) p_{i j}(s) x_{j}(s) d s\right| \\
& \leq \sum_{j} \int_{\alpha}^{\beta} g(t, s, a, b)\left|p_{i j}(s)\right|\left|y_{j}(s)\right|\left|x_{j}(s)\right|\left|y_{j}^{-1}(s)\right| d s \\
& \leq \sum_{j} \int_{a}^{b} g(t, s, a, b)\left|p_{i j}(s)\right|\left|y_{j}(s)\right| d s\|x\| \\
& <\sum_{j} \int_{a}^{b} g(t, s, a, b)\left|q_{i j}(s)\right|\left|y_{j}(s)\right| d s\|x\|
\end{aligned}
$$

where the very last inequality is a strict inequality because of the hypothesis relating $p_{i j}$ and $q_{i j}$, the fact from Lemma 2 that none of the $q_{i j}$ are zero, the fact that none of the $y_{j}$ vanish on $(a, b)$, and that $g(t, s, a, b)$ does not vanish for $s$ on $(a, b)$. Thus for $i=1, \ldots, m$,

$$
\frac{\left|x_{i}(t)\right|}{\left|y_{i}(t)\right|}<\frac{1}{\left|y_{i}(t)\right|} \sum_{j} \int_{a}^{b} g(t, s, a, b)\left|q_{i j}(s)\right|\left|y_{j}(s)\right| d s\|x\| .
$$

Of course if $[\alpha, \beta] \subset(a, b)$, then (4) extends to a strict inequality on $[\alpha, \beta]$, since in this case $y_{i} \neq 0$ on $[\alpha, \beta]$. If $\alpha=a$ or $\beta=b$, there remains a problem. However, it will now be shown that if $\alpha=a$, (4) holds as a strict inequality when $t=a^{+}$. To show this one needs to show that the following strict inequality given by

$$
\begin{aligned}
\sum_{j} \int_{a}^{b} g(t, s, a, b)\left|p_{i j}(s)\right|\left|y_{j}(s)\right| d s /\left|y_{i}(t)\right| \\
<\sum_{j} \int_{a}^{b} g(t, s, a, b)\left|q_{i j}(s)\right|\left|y_{j}(s)\right| d s /\left|y_{i}(t)\right|
\end{aligned}
$$


remains a strict inequality as $t \rightarrow a^{+}$if $\alpha=a$. This can be seen by realizing that the limit as $t \rightarrow a^{+}$of the left-hand side of (5) is just

$$
\sum_{j} \int_{a}^{b} \frac{\partial^{k}}{\partial t^{k}} g(a, s, a, b)\left|q_{i j}(s)\right|\left|y_{j}(s)\right| d s / y_{i}^{(k)}(a) \mid
$$

by L'Hôpital's rule and the limit as $t \rightarrow a^{+}$of the right-hand side of (5) is

$$
\sum_{j} \int_{a}^{b} \frac{\partial^{k}}{\partial t^{k}} g(a, s, a, b)\left|p_{i j}(s)\right|\left|y_{j}(s)\right| d s /\left|y_{i}^{(k)}(a)\right| .
$$

Recalling that $\left(\partial^{k} / \partial t^{k}\right) g(a, s, a, b)$ is positive on $(a, b)$, it follows readily as before that (6) is strictly less than (7). A similar argument is used for $t \rightarrow b^{-}$if $\beta=b$.

It therefore follows that

(8) $\left\|x_{i}\right\|<\sup _{t \in(\alpha, \beta)} \sum_{j} \int_{a}^{b} g(t, s, a, b)\left|q_{i j}(s)\right|\left|y_{j}(s)\right| d s\|x\| /\left|y_{i}(t)\right|$

for $i=1, \ldots, m$.

For $t \in(a, b), y(t) \in K^{\circ}, y_{i}(t)=\left|y_{i}(t)\right| \delta_{i}$ and $y_{i}(t) \neq 0$. Also, from Lemma 2, $\left|q_{i j}(s)\right|=q_{i j}(s) \delta_{i} \delta_{j}$ and $q_{i j}(s) \neq 0$. Thus $\left|y_{i}(t)\right|=y_{i}(t) \delta_{i}$ and

$$
\begin{aligned}
& \sum_{i} \int_{a}^{b} g(t, s, a, b)\left|q_{i j}(s)\right|\left|y_{j}(s)\right| d s \\
& \quad=\delta_{i} \sum_{j} \int_{a}^{b} g(t, s, a, b) q_{i j}(s) y_{j}(s) d s=\delta_{i} y_{i}(t) .
\end{aligned}
$$

Therefore the right-hand side of (8) is just $\|x\|$, i.e., $\left\|x_{i}\right\|<\|x\|$ for $i=1, \ldots, m$. This in turn implies that $\|x\|<\|x\|$. From this contradiction, we infer the truth of the theorem.

The following example shows that the condition $p_{i j}(t) \leq\left|q_{i j}(t)\right|$ is not sufficient to obtain the conclusion of Theorem 2 . Let $q$ be the identity matrix and let $p=$ $\left[\begin{array}{cc}1 & -1 \\ -1 & 1\end{array}\right], n=2$ and $k=1$. Of course $c_{q}(0)=\pi$. Notice that two solutions of $x^{\prime \prime}+p x=0$ are $x=(\sin \sqrt{2} t,-\sin \sqrt{2} t)$ and $x=(t, t)$. Thus $c_{p}(0)=\pi / \sqrt{2}<c_{q}(0)$. This examples also shows that $p_{i j}(t) \leq q_{i j}(t)$ does not imply $c_{q}(0) \leq c_{p}(0)$.

Notice furthermore that if $q=\left[\begin{array}{cc}1 & -1 \\ -1 & 1\end{array}\right]$ and $p$ is the identity matrix, Theorem 2 would apply and show that $c_{p}(0)>\pi / \sqrt{2}$, but the classical comparison theorem of Morse would not apply here since neither $p-q$ nor $q-p$ are positive semidefinite.

\section{REFERENCES}

1. S. Ahmad and A. C. Lazer, On the components of extremal solutions of second order systems, SIAM Math. Anal. 8 (1977), 16-23.

2. class of nonselfadjoint systems, SIAM Math. Anal. 9 (1978), 1137-1150.

3. S. Cheng, Nonoscillatory solutions of $x^{(m)}=(-1)^{m} Q(t) x$, Canad. Math. Bull. 22 (1979), 17-21.

4. M. Keener and C. Travis, Focal points and positive cones for a class of $n$th order differential equations, Trans. Amer. Math. Soc. 237 (1978), 331-351. 
5. __ Sturmian theory for a class of nonselfadjoint differential systems, Ann. Mat. Pura Appl. 123 (1980), 247-266.

6. M. Morse, $A$ generalization of the Sturm separation and comparison theorems in $n$-space, Math. Ann. 103 (1930), 53-69.

7. Z. Nehari, Green's functions and disconjugacy, Arch. Rational Mech. Anal. 62 (1976), 53-76.

8. W. T. Reid, Ordinary differential equations, Wiley, New York, 1971.

9. K. Schmitt and H. L. Smith, Positive solutions and conjugate points for systems, Nonlinear Anal. 2 (1978), 93-105.

10. H. L. Smith, A note on disconjugacy of second order systems, Pacific J. Math. 89 (1980), 447-452.

11. E. Tomastik, Comparison theorems for second order nonselfadjoint differential systems, SIAM Math. Anal. 14 (1983), 60-65.

12. C. Travis, Comparison of eigenvalues for linear differential equations of order $2 n$, Trans. Amer. Math. Soc. 177 (1973), 363-374.

Department of Mathematics, University of CONnecticut, Storrs, ConNECTICUT 06268 